\title{
Detection of Biofilm Formation and Antibiotic Resistance in Klebsiella Oxytoca and Klebsiella Pneumoniae from Animal Origin Foods
}

\author{
Gedif Meseret Abebe ${ }^{1,2}$ \\ ${ }^{1}$ Department of Biology, College of Natural and Computational Science, Wolaita Sodo University, Wolaita Sodo, Ethiopia \\ ${ }^{2}$ Department of Biology, School of Natural and Applied Science, Gazi University, Ankara, Turkey
}

Email address:

gedif.meseret@wsu.edu.et, gedifm@gmail.com

\section{To cite this article:}

Gedif Meseret Abebe. Detection of Biofilm Formation and Antibiotic Resistance in Klebsiella Oxytoca and Klebsiella Pneumoniae from Animal Origin Foods. International Journal of Microbiology and Biotechnology. Vol. 5, No. 3, 2020, pp. 120-130.

doi: $10.11648 /$ j.ijmb.20200503.17

Received: May 17, 2020; Accepted: May 29, 2020; Published: July 4, 2020

\begin{abstract}
Biofilms are surface-attached microbial communities with distinct properties, which have a tremendous impact on our health and food safety. The study was aimed to detect biofilm formation and antibiotic resistance by Klebsiella oxytoca and Klebsiella pneumoniae from animal origin foods. In this study100 food samples were examined for the presence of Klebsiella oxytoca, Klebsiella pneumoniae, and other Enterobacteriaceae family members. In this study, 19 Klebsiella oxytoca and 5 Klebsiella pneumoniae isolates were isolated from cheese and minced meat samples using standard biochemical tests and identification kit. Biofilm formation in these isolates was detected by using microplate, Congo red agar, and tube adherence methods. Antibiotic susceptibility testing was performed using the disk diffusion method on Muller-Hinton agar. Using a microplate method strong biofilm formation was observed in $16(84 \%)$ and $5(100 \%)$ isolates of Klebsiella oxytoca and Klebsiella pneumoniae after 24 hours of incubation on Tryptic Soy Broth medium containing $2 \%$ of glucose respectively. After 24 and 48 hours of incubation on Tryptic Soy Broth without glucose strong biofilm formation was detected in 10 (52.6\%) and $2(40 \%)$ isolates of Klebsiella oxytoca and Klebsiella pneumoniae respectively. After 24 hours of incubation on Congo red agar $14(73.7 \%)$ and $3(60 \%)$, isolates of Klebsiella oxytoca and Klebsiella pneumoniae were slime factor positive respectively. In tube adherence method $13(68.4 \%)$ and $4(80 \%)$, isolates of Klebsiella oxytoca and Klebsiella pneumoniae were seen to have adhered strongly after 24 hours of incubation on Tryptic Soy Broth medium containing 2\% of glucose. In general, strong biofilm formation by these strains was seen on Tryptic Soy Broth medium when supplemented with glucose. Among all the Klebsiella oxytoca, the highest rates of susceptibility were seen toward Trimethoprim-Sulfamethoxazole (100\%) and Imipenem (94.7\%) followed by Chloramphenicol (73.7\%) and gentamicin (68.4\%). Among 19 Klebsiella oxytoca isolates, the highest rates of resistance were seen in streptomycin (73.7\%) and Kanamycin (73.7\%) followed by ampicillin (63.2\%). The majority of Klebsiella pneumoniae isolates were resistant to Kanamycin (80\%) and Streptomycin (80\%) followed by Amikacin $(60 \%)$. On the other hand, $80 \%$ of Klebsiella pneumoniae were susceptible to imipenem, chloramphenicol, TrimethoprimSulfamethoxazole, and cefotaxime. Generally, majority of Klebsiella oxytoca, and Klebsiella pneumoniae isolates showed strong biofilm production on different growth conditions and majority of the isolates were also resistance for antibiotics. Therefore, biofilm production by these nosocomial bacteria has an implication public health and pave the way for increased resistance of biofilm-associated organisms to antimicrobial agents.
\end{abstract}

Keywords: Klebsiella oxytoca, Klebsiella pneumoniae, Biofilm, Antibiotic Resistance

\section{Background}

Klebsiella species are gram-negative bacteria that can cause hospital-acquired infection. It has been reported that
Klebsiella spp accounts for $8 \%$ of all nosocomial infections in the United States of America and Europe [1]. Klebsiella oxytoca is an emerging bacterial isolate causing nosocomial infection in adults, and having multiple drug resistance to 
most of the antibiotics used for the treatment of bacterial infections [2, 3]. As Zollner-Schwetz et al., reported $\mathrm{K}$. oxytoca is also an intestinal pathobiont and the causative agent of hemorrhagic colitis [4]. Nosocomial Klebsiella infections are also caused mainly by Klebsiella pneumoniae, the medically most important species of the genus. Similarly, Klebsiella pneumoniae is a pathogenic bacterium that causes infections due to its high virulence factor and general occurrence of resistance to most antibiotics [5, 6]. Klebsiella pneumoniae is rod-shaped bacterium with a prominent capsule, mucoid colonies, hospital-acquired, opportunistic pathogen that can cause bacteremia, urinary and respiratory tract infections and especially in immunosuppressed patients whose natural host defense fails [1]. In addition to clinical samples, Klebsiella pneumoniae is also found in the environment and as a harmless commensal. It is also a frequent nosocomial pathogen (causing urinary, respiratory and blood infections) and the agent of specific human infections [7]. Besides nosocomial infection Klebsiella pneumoniae is also an important foodborne pathogen that can cause septicaemia, liver abscesses, and diarrhea in humans. $\mathrm{K}$. pneumonia contamination was recorded in $32 \%$ of the street food samples examined in Malaysia [8]. This bacterium is also an issue of domestic animals that threatens livestock production as well as scourging public health since these animals could serve as a vehicle for multidrug-resistant K. pneumoniae strains [9].

Biofilms are complex microbial communities with unique properties, which have an impact on food safety and our health. Biofilm can be defined as a microbial community that is attached irreversibly to biotic and abiotic surfaces and encased by a self-produced matrix of extracellular polymeric substances [10]. Therefore, biofilms are a self-protection growth pattern of bacteria from hostile conditions, which are different from planktonic counterparts. Since they firmly attached to the different food surfaces, they will be a source of contamination and hence, in turn, reduce the quality and safety of food. They have been of considerable interest in food hygiene since biofilms may contain spoilage and pathogenic bacteria which increases post-processing contamination and risk to public health [11]. Besides, reducing the quality and safety of the foods, biofilm is also causing health problems for the patients with indwelling medical devices via attachment of cells to the surface matrix [12]. Since microbes produce extracellular polysaccharide matrices that have the potential to physically prevent access to certain antimicrobial agents or antibiotics, they become troublesome in different sectors. Bacteria within this matrix are tolerance to antibiotics and the host immune system compared to growth as planktonic cells [13]. Biofilm formation by K. pneumoniae was found to form characteristic biofilms on the substratum $[13,14]$. The ability of bacteria to form biofilms on medical devices, such as catheters, has a major role in the development of many nosocomial infections [13]. Biofilms have an effect on sanitizers, cause economic losses to industries, and contaminate food and can increase the level of antimicrobial resistance.
Biofilm formation does not only confer survival ability to the pathogen in the human hosts, but also persistence in the fluctuating environment such as variable $\mathrm{pH}$, temperature, carbon sources, and fluid flow $[15,16]$. Biofilm forming bacteria are more resistant antibacterial agents than their counter part free-living planktonic cells. Ribeiro et al reported that infections caused by biofilm-forming Klebsiella pneumoniae are more difficult to treat when compared to planktonic Klebsiella pneumoniae infections [17]. Biofilm formation allows microbes to be resistant to sanitizers and prolonged existence in the manufacturing and food environments. Microbes found in the biofilm can detach or slough off into foods and can be a source of foodborne infection. As a number of reports revealed the attachment and prolonged persistence of biofilm-forming foodborne pathogens on food contact surfaces become sources of contamination that threaten quality and safety of food products, and in turn leads serious hygienic problems and economic losses [18, 19]. This surface attached microbial community is resistant to disinfectant or sanitizers since they are encased by matrix [20]. As most studies revealed the presence of biofilms in the food systems is a serious public health risk.

Antimicrobial resistance is an ever-growing and serious public health problem that is rapidly increasing across the world which contributes to an increment of morbidity and mortality especially in developing countries [21]. Overdose utilization of antibiotics and disinfectants in different sectors such as in clinical, agriculture, and fish culture and disposing of them without treatment into the environment creates conducive conditions for the emergence of multidrugresistant pathogenic bacteria. Polymicrobial infection and immuno-compromised accompanied by exposures to multiple antibiotics increase the risks of infections and drug resistance. Klebsiella pneumoniae is an escalating and major cause of hospital infections in humans and showed multidrug-resistant (MDR) which increases morbidity and mortality [22]. Many new resistant strains of Klebsiella pneumoniae, Enterobacter cloacae, and Escherichia coli have emerged as significant hospital-acquired pathogens. As Adamo and Margarit reported Klebsiella pneumoniae is one of the newly threatening pathogens, is particularly worrisome in the nosocomial setting, and its surface polysaccharides are regarded as promising antigen candidates [23].

Understanding the mechanisms of biofilm formation and antibiotic resistance by Klebsiella pneumoniae and Klebsiella oxytoca would help to design controlling strategies and intervention mechanisms of biofilm formation. Therefore, the aim of this study was to detect biofilm formation and antibiotic resistance by Klebsiella pneumoniae and Klebsiella oxytoca isolated from cheese, and minced meat samples.

\section{Materials and Methods}

\subsection{Collection of Samples}

In this study a total of 100 cheese and minced meat food 
samples were collected from super market in Ankara. The samples were collected and placed in separate sterile plastic bags and then transported to the laboratory using ice packs and processed within two hours for microbial analysis.

\subsection{Isolation and Identification of Klebsiella Spp}

About $5 \mathrm{~g}$ of each sample was added and homogenized with $45 \mathrm{ml}$ buffered peptone water (BPW) and incubated at $37^{\circ} \mathrm{C}$ for 24 hours. After pre-enrichment, $10 \mathrm{ml}$ of preenriched sample was used to inoculate $90 \mathrm{ml}$ of Enterobacteriaceae enrichment broth and incubated at $37^{\circ} \mathrm{C}$ for $24 \mathrm{~h}$. After incubation, a loopful of enriched culture was streaked on VRBG (Violet Red Bile Glucose) agar surface and the plates are incubated at $37^{\circ} \mathrm{C}$ for $24 \mathrm{~h}$ and another 0.1 $\mathrm{ml}$ of the same culture was taken and spread onto VRBGA agar plates and incubated for $24 \mathrm{~h}$ at $37^{\circ} \mathrm{C}$. Typical pink, mucoid colonies on VRBG agar were streaked on TSA (Tryptic Soya Agar) and subjected to further characterization using biochemical and BBL crystal identification kit.

\subsection{Detection of Biofilm Formation by Klebsiella spp}

\subsubsection{Detection of Biofilm by Microtiter Plate Method}

Quantification of biofilm production by microtiter plate method was performed according to Stepanovi'c et al. [24]. $24 \mathrm{Klebsiella} \mathrm{spp} \mathrm{isolates} \mathrm{were} \mathrm{grown} \mathrm{overnight} \mathrm{at} 37^{\circ} \mathrm{C}$ as pure cultures on Tryptone Soya agar. Groups of three single colonies were inoculated in $5 \mathrm{~mL}$ Tryptone Soya broth. Suspensions were incubated for $24 \mathrm{~h}$ at $37^{\circ} \mathrm{C}$ and then diluted at $1 / 100$ ratio with fresh TSB and TSB containing $2 \%$ glucose. This dilution was used as the inoculum and for each Klebsiella spp, $200 \mu \mathrm{L}$ aliquots of prepared suspension were inoculated into three wells of the 96 microtiter plates. The negative control wells contained broth only: then the plates were incubated at $37^{\circ} \mathrm{C}$ for $24 \mathrm{~h}$. After incubation, the microtiter plates content of each well was removed by tapping the bottom plates. The wells were washed with $300 \mu \mathrm{L}$ of phosphate buffer saline to remove free-floating bacteria. Biofilms formed by bacteria adherent to the wells were fixed by $99 \%$ methanol for 20 minutes and stained with $1 \%$ crystal violet (CV) for 15 minutes [24]. Plates containing excess stain were washed and kept for drying. After the microplates were air-dried, the dye bound to the adherent cells were released with $150 \mu \mathrm{l}$ of $33 \%(\mathrm{v} / \mathrm{v})$ glacial acetic acid and left for at least 30 minutes at room temperature. Then the optical density of the stained adherent biofilm was measured using a micro- ELISA auto-reader at a wavelength of $570 \mathrm{~nm}$. For each strain the experiment was conducted in triplicate and repeated three times. Interpretation of biofilm production was performed based on the criteria described by Stepanovic et al. [24]. Isolates were interpreted and categorized as follow. 1. nonbiofilm producers (-) (OD $\leq$ ODc); (2) weak biofilm producers $(+)(\mathrm{ODc}<\mathrm{OD} \leq 2 \times$ ODc); (3) moderate biofilm producers $(++)(2 \times$ ODc $<$ OD $\leq$ $4 \times$ ODc $)$; (4) Strong biofilm producers $(+++)(4 \times$ ODc $<$ OD). Optical density cut-off value (ODc) is defined as three standard deviations above the mean OD of the negative control $[24,25]$

\subsubsection{Biofilm Detection by Tube Adherence}

Tube adherence method is also another method to detect biofilm formation. In this method a loopful of Klebsiella spp incubated on TSA was taken and inoculated in $2 \mathrm{~mL}$ of trypticase soy broth with $2 \%$ glucose in test tubes and with another $2 \mathrm{~mL}$ of Tryptic Soy Broth without glucose. The tubes with $2 \%$ glucose and without glucose were incubated at $37 \mathrm{oC}$ for $24 \mathrm{~h}$. Then after the contents inside the tubes were decanted and washed with phosphate buffer saline and dried. Tubes were then stained with crystal violet (1\%) and left for 15 minutes. After that the stained tubes were dried in inverted position. The formation of visible line on the walls of test tube was regarded as positive for biofilm formation. The amount of biofilm formed was scored as none (-), weak $(+)$, moderate $(++)$ and strong $(+++)$. The experiment was performed in triplicate and repeated three times [26].

\subsubsection{Detection of Slime Production by Congo Red Agar Method}

Biofilm formation was also detected using the third method called Congo Red Agar Method. According to this method the prepared CRA plates were inoculated with Klebsiella spp and incubated at $37^{\circ} \mathrm{C}$ for 24 hours. After incubation, the result was judged based on colony color and morphology. Therefore, black colonies with a dry crystalline consistency indicated biofilm production. Red colonies with smooth, round, and shiny surface were indicative of negative slime production [27]

\subsection{Antibiotic Susceptibility Testing}

Antimicrobial susceptibility was performed by the KirbyBauer disc diffusion technique following standard procedures on Muller-Hinton agar (Oxoid, Basings toke, UK). Klebsiella spp were tested for susceptibility to ampicillin (10 $\mu \mathrm{g})$, trimethoprim-sulfamethoxazole $(1.25 / 23.75 \mu \mathrm{g})$, cefotaxime $(30 \mu \mathrm{g})$, tobramycin $(10 \mu \mathrm{g})$, nalidixic acid $(30$ $\mu \mathrm{g})$, amikacin $(30 \mu \mathrm{g})$, chloramphenicol $(30 \mu \mathrm{g})$, tetracycline (30 $\mu \mathrm{g})$, gentamycin $(10 \mu \mathrm{g})$, kanamycin $(10 \mu \mathrm{g})$, and streptomycin $(10 \mu \mathrm{g})$. All antibiotics tested were included in a list of antibiotics for susceptibility test of Enterobacteriaceae in National Committee for Clinical Laboratory Standards. Zone of inhibition was measured, compared and scored as sensitive, intermediate, and resistant according the criteria set by National Committee for Clinical Laboratory Standards [28].

\section{Results}

A total of 24 Klebsiella spp were isolated from cheese and minced meat samples analyzed. Out of 24 Klebsiella spp, 19 (79\%) isolates were Klebsiella oxytoca and the rest 5 (21\%) isolates were Klebsiella pneumoniae. Majority of Klebsiella oxytoca, $16(80 \%)$ were isolated from cheese samples and the rest were isolated from minced meat samples. Similarly 4 (20\%) Klebsiella pneumonia was isolated from cheese 
samples and the rest was isolated from minced meat [Table 1]. In addition to Klebsiella spp other Enterobacteriaceae family members were also detected in these animal origin food samples. From 100 food samples analyzed 24 were positive for Klebsiella oxytoca and Klebsiella pneumonia and the rest were members of Enterobacteriaceae family.

Table 1. Klebsiella spp. isolated from cheese and minsced meat samples.

\begin{tabular}{llll}
\hline Types of & Number of positive & Klebsiella species & \\
\hline food sample & samples & Klebsiella oxytoca & $\begin{array}{l}\text { Klebsiella } \\
\text { pneumonia }\end{array}$ \\
\hline $\begin{array}{l}\text { Cheese } \mathrm{n}=50 \\
\text { Minced meat } \\
\mathrm{n}=50\end{array}$ & $20(40 \%)$ & $16(80 \%)$ & $4(20 \%)$ \\
\hline
\end{tabular}
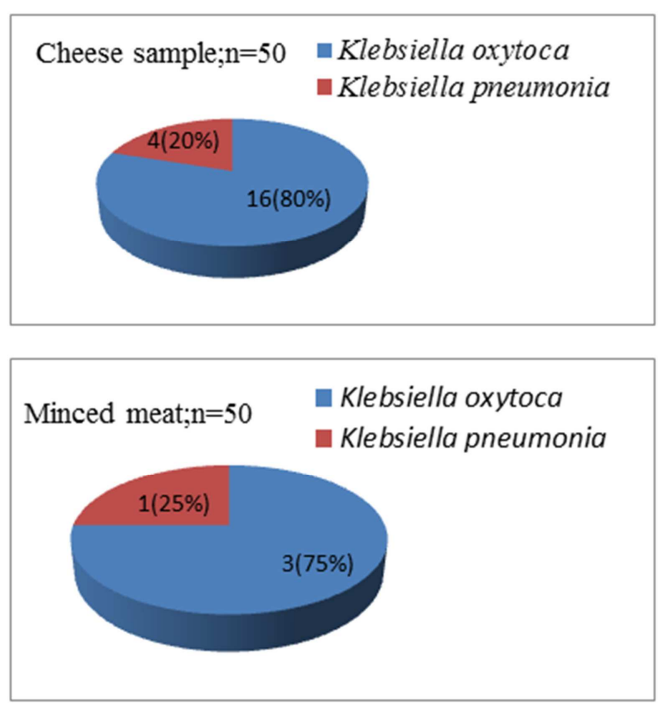

Figure 1. Klebsiella spp. isolated from cheese and minced meat samples.

\subsection{Detection of Biofilm Formation by Microtiter Plate Method}

As shown on figure 1 and table 2 majority of Klebsiella species were positive for biofilm production with Microtiter Plate Method. As indicated on table 1, out of 19 Klebsiella oxytoca isolates $16(84 \%)$ were strong biofilm producers on TSB supplemented with $2 \%$ glucose after 24 hours of incubation. The rest $3(16 \%)$ Klebsiella oxytoca isolates produce biofilm moderately on the same media and growth conditions. Similarly $12(63 \%)$ isolates show strong biofilm production with slight variation within the same medium after 48 hours of incubation. In TSB medium without glucose after 24 hours of incubation 52.6\% (10/19), 36.6\% (6/19) and $10.5 \%(2 / 19)$ of Klebsiella oxytoca isolates were strong, moderate and weak biofilm producers respectively. But biofilm production was not seen one strain. On the same TSB medium after 48 hours of incubation, out of 19 Klebsiella oxytoca isolates, $21.1 \%(4 / 19), 47.4 \%(9 / 19)$ and $26.3 \%$ $(5 / 19)$, and $5.3 \%(1 / 19)$ were strong, moderate, weak and negative biofilm producers respectively [Table 2] [Figure 2]. After 24 hours of incubation all Klebsiella pneumonia isolates were strong biofilm producers on TSB medium with $2 \%$ glucose. After 48 hours of incubation $40 \%(2 / 5), 40 \%$ $(2 / 5)$, and 20 (1/5) Klebsiella pneumonia isolates show strong, moderate and weak biofilm production. Some variation was also seen when these isolates were grown on TSB medium without glucose. Out of all isolates of Klebsiella pneumoniae, $20 \%(2 / 5)$ and $40 \%(3 / 5)$ of them produce strong and moderate biofilms after 24 hours of incubation. Slight variation was also seen when these isolates are incubated for 48 hours on the same medium [Table 2] [Figure 3].

Table 2. Detection of biofilm formation by Klebsiella oxytoca and Klebsiella pneumoniae using Microtiter Plate Method.

\begin{tabular}{|c|c|c|c|c|c|c|c|c|}
\hline & \multicolumn{4}{|c|}{ Klebsiella oxytoca } & \multicolumn{4}{|c|}{ Klebsiella pneumonia } \\
\hline & & \multicolumn{7}{|c|}{ growth medium } \\
\hline \multicolumn{3}{|l|}{ Biofilm } & \multicolumn{6}{|c|}{ incubation hours } \\
\hline formation & \multicolumn{2}{|c|}{ TSB+2\% glucose } & TSB & & \multicolumn{2}{|c|}{ TSB+2\%glucose } & \multicolumn{2}{|l|}{ TSB } \\
\hline n (\%) & 24 hours & 48 hours & 24 hours & 48 hours & 24 hours & 48 hours & 24 hours & 48 hours \\
\hline Strong & $16(84 \%)$ & $12(63 \%)$ & $10(53 \%)$ & $4(21 \%)$ & $5(100 \%)$ & $2(40 \%)$ & $2(40 \%)$ & $1(20 \%)$ \\
\hline Moderate & $3(16 \%)$ & $6(32 \%)$ & $6(32 \%)$ & $9(47 \%)$ & 0 & $2(40 \%)$ & $3(60 \%)$ & $1(20 \%)$ \\
\hline Weak & 0 & $1(5 \%)$ & $2(11 \%)$ & $5(26 \%)$ & 0 & $1(20 \%)$ & 0 & $2(40 \%)$ \\
\hline Negative & 0 & 0 & $1(5 \%)$ & $1(5 \%)$ & 0 & 0 & 0 & $1(20 \%)$ \\
\hline
\end{tabular}

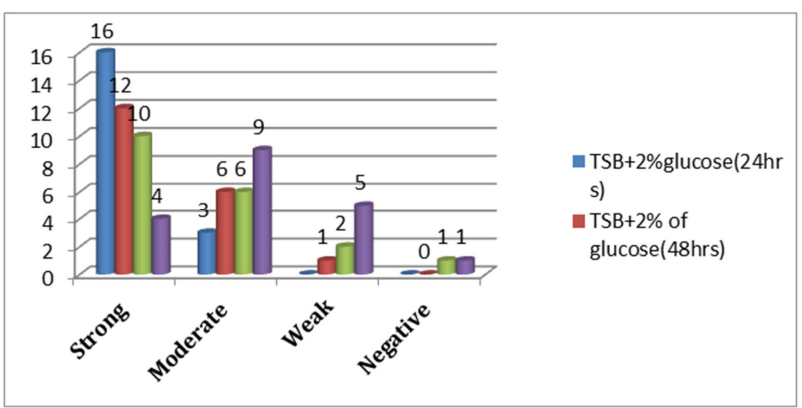

Figure 2. Detection of biofilm formation by Klebsiella oxytoca using Microtiter Plate Method.

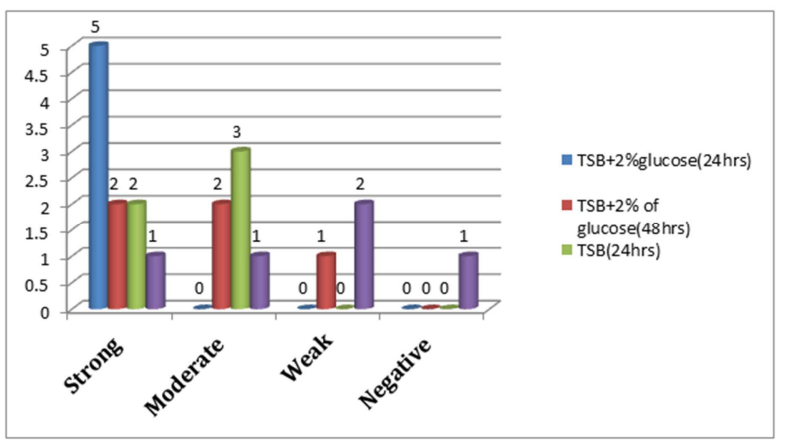

Figure 3. Detection of biofilm formation by Klebsiella pneumoniae using Microtiter Plate Method. 
According to the results reported in Table 2 and figure 2 majority of Klebsiella oxytoca isolates produced strong biofilm after 24 hours of incubation on TSB medium supplemented with $2 \%$ of glucose. Similarly with slight difference strong biofilm formation was detected after 48 hours of incubation on similar medium [Figure 2].

As shown on figure 3 Klebsiella pneumoniae also showed strong biofilm formation TSB supplemented $2 \%$ of glucose within 24 hours of incubation and nearly similar biofilm formation was detected after 48 hours of incubation on same medium [Figure 3].

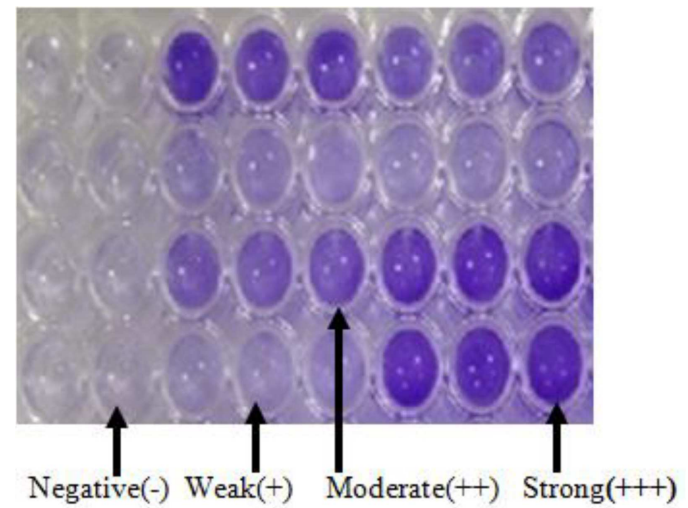

As shown on figure 4 the level of biofilm production was designated as strong, moderate, weak and negative, which was confirmed based on optical density (OD) value using ELISA.

\subsection{Detection of Biofilm Formation by Klebsiella Oxytoca and Klebsiella Pneumoniae by Tube Adherence Method}

Using tube method on TSB medium supplemented with $2 \%$ of glucose, out of 19 Klebsiella oxytoca isolates $68 \%$ $(13 / 19)$ and $16 \%(3 / 19)$ were strong and moderate biofilm producers after 24 hours of incubation respectively. On the same incubations periods in TSB without adding glucose $26 \%(5 / 19), 47 \%(9 / 19), 5 \%(1 / 19)$ and $21 \%(4 / 19)$ were strong, moderate, weak and negative biofilm producers respectively. Majority of Klebsiella pneumonia isolates were strong biofilm producers on TSB medium supplemented with $2 \%$ of glucose and variation on biofilm production was seen was on TSB medium without glucose. As shown on figure 6 biofilm formations was considered positive when there is a visible film lined on the wall and the bottom of the tube [Table 3].

Figure 4. Microtiter plate method showing strong, moderate, and weak and none biofilm producers based on crystal violet stain.

Table 3. Detection of biofilm formation by Klebsiella oxytoca and Klebsiella pneumonia using CRA and tube method.

\begin{tabular}{|c|c|c|c|c|c|c|}
\hline & Tube method & & & & CRA & \\
\hline Biofilm & Klebsiella oxytoca & & Klebsiella pneumoni & & Klebsiella oxytoca & Klebsiella pneumonia \\
\hline formation & growth medium & & growth medium & & growth medium & growth medium \\
\hline n (\%) & incubation hours & & incubation hours & & incubation hours & incubation hours \\
\hline & TSB $+2 \%$ glu & TSB & TSB $+2 \%$ glu & TSB & CRA & CRA \\
\hline & 24hours & 24 hours & 24 hours & 24 hours & 24 hours & 24 hours \\
\hline Strong & $13(68 \%)$ & $5(25 \%)$ & $4(80 \%)$ & $1(20 \%)$ & $14(74 \%)$ & $3(60 \%)$ \\
\hline Moderate & $3(16 \%)$ & $9(47 \%)$ & $1(20 \%)$ & $4(80 \%)$ & $3(16 \%)$ & $2(40 \%)$ \\
\hline Negative & $3(16 \%)$ & $4(21 \%)$ & 0 & 0 & $1(5 \%)$ & 0 \\
\hline
\end{tabular}

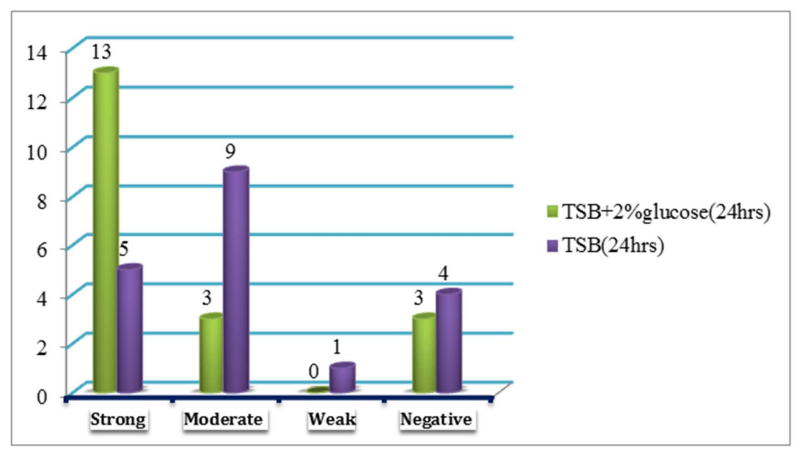

Figure 5. Detection of biofilm formation by Klebsiella oxytoca using tube adherence method.

Similarly, Klebsiella oxytoca also strong biofilm production using tube adherence methods. As shown on figure 5 majority of the Klebsiella oxytoca isolates were strong biofilm producers when supplemented with $2 \%$ of glucose. There were also strains that produced strong biofilm on TSB medium without glucose. Few strains were also negative on both growth media [Figure 5].

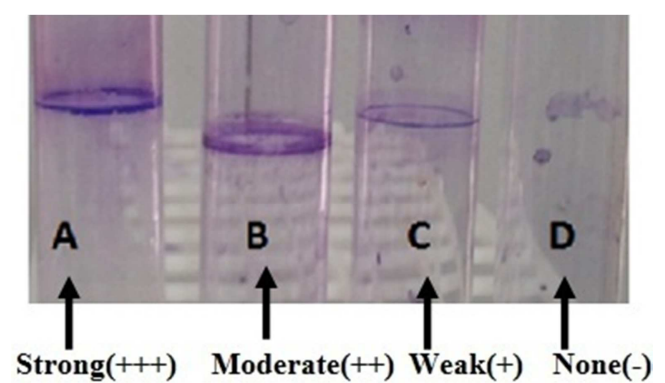

Figure 6. Detection of biofilm formation by Klebsiella spp using tube method.

\subsection{Detection of Slime Production by Congo Red Agar Method}

In our study, slime production was examined depending on colony morphology of Klebsiella spp produced on Congo red 
agar. Using Congo red agar method out of 19 Klebsiella oxytoca, 74\% (14/19), 16\% (3/19), 5\% (1/19) and 5\% (1/19) were strong, moderate, weak and negative biofilm producers after 24 hours of in incubation respectively. As shown on table 2 within 24 hours of incubation $60 \%$ and $40 \%$ of Klebsiella pneumonia were strong and moderate biofilm producers respectively. Black colonies which indicate biofilm production and red colonies which are indicative of negative slime production are showed in figure 7.

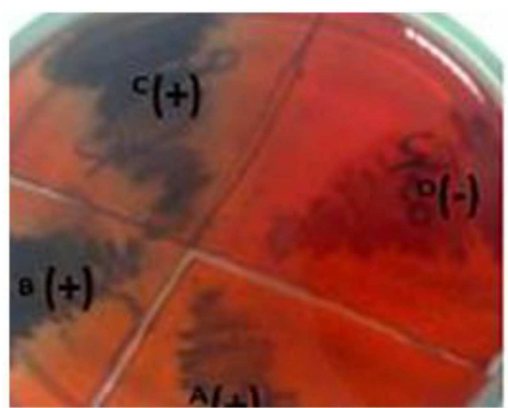

Slime factor positive $(\mathrm{A}, \mathrm{B}, \mathrm{C})$ and Slime factor negative (D)

Figure 7. Detection of biofilm (slime factor) producer Klebsiella spp using Congo red agar.

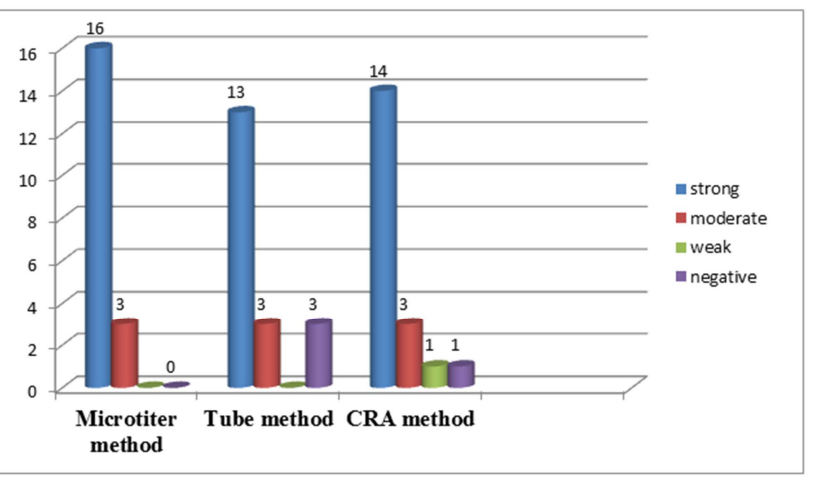

Figure 8. Detection of biofilm formation by Klebsiella oxytoca using microtiter, tube adherence and CRA method.

As figure 8 revealed majorities of Klebsiella oxytoca isolates produced strong biofilms and almost similar result were seen and confirmed using the three biofilm detection methods.

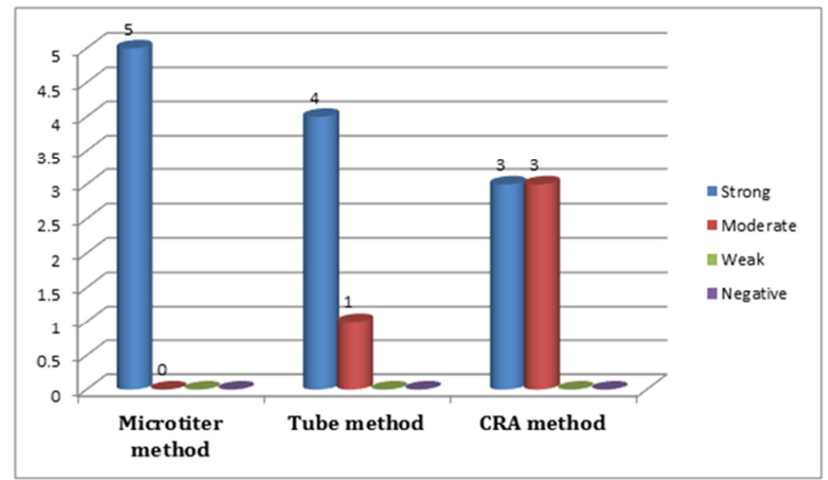

Figure 9. Detection of biofilm formation by Klebsiella pneumoniae using microtiter, tube adherence and CRA method.

As figure 9 revealed majority of Klebsiella pneumoniae isolates produced strong biofilms and almost very close result was obtained using the three methods i.e microtiter, tube adherence and CRA method

\subsection{Antimicrobial-Resistance Profile of Klebsiella Species Isolates}

Among the 19 Klebsilla oxytoca isolates studied, 14 (73.7\%) were resistant to Streptomycin, $14(73.7 \%)$ to Kanamycin, $8(42 \%)$ to Amikasin, $5(26.3 \%)$ to Tobramysin, $4(21.1 \%)$ to Nalidixic acid, $4(21.1 \%)$ to Tetracycline, 2 (10.5\%) to Gentamycin, 2 (10.5\%) to Cloramphenicol and 1 (5.3\%) to Cefotaxime. Among the 5 Klebsiella pneumoniae isolates studied, $4(80 \%)$ were reistant to Streptomycin, 4 $(80 \%)$ to Kanamisin, $3(60 \%)$ to Amikasin and $1(20 \%)$ to Tobramysin [Table 3].

Table 4. Antimicrobial-Resistance Profile of Klebsiella spp. isolates.

\begin{tabular}{|c|c|c|c|c|c|c|}
\hline \multirow{3}{*}{ Antibiotics } & \multicolumn{3}{|c|}{ Klebsilla oxytoca $(\mathrm{n}=19)$} & \multicolumn{3}{|c|}{ Klebsiella pneumoniae $(\mathrm{n}=5)$} \\
\hline & \multicolumn{3}{|c|}{ Number of isolates (\%) } & \multicolumn{3}{|c|}{ Number of isolates (\%) } \\
\hline & $\mathbf{S}$ & I & $\mathbf{R}$ & $\mathbf{S}$ & I & $\mathbf{R}$ \\
\hline Ampicillin & $6(31.6 \%)$ & $1(5.3 \%)$ & $12(63.2 \%)$ & 0 & $2(40 \%)$ & $3(60 \%)$ \\
\hline Trimethoprim-sulfamethoxale & $19(100 \%)$ & 0 & 0 & $4(80 \%)$ & $1(20 \%)$ & 0 \\
\hline Cefotaxime & $9(47.4 \%)$ & $8(42 \%)$ & $1(5.3 \%)$ & $4(80 \%)$ & $1(20 \%)$ & 0 \\
\hline Tobramycin & $8(42 \%)$ & $6(31.6 \%)$ & $5(26.3 \%)$ & $1(20 \%)$ & $3(60 \%)$ & $1(20 \%)$ \\
\hline Nalidixic acid & $6(31.6 \%)$ & $9(47.4 \%)$ & $4(21.1 \%)$ & $2(40 \%)$ & $3(60 \%)$ & 0 \\
\hline Amikacin & $6(31.6 \%)$ & $5(26.3 \%)$ & $8(42 \%)$ & $1(20 \%)$ & $1(20 \%)$ & $3(60 \%)$ \\
\hline Chloramphenicol & $14(73.7 \%)$ & $3(15.8 \%)$ & $2(10.5 \%)$ & $2(40 \%)$ & $3(60 \%)$ & 0 \\
\hline Tetracycline & $10(52.6 \%)$ & $5(26.3 \%)$ & $4(21.1 \%)$ & $3(60 \%)$ & $2(40 \%)$ & 0 \\
\hline Gentamycin & $13(68.4 \%)$ & $4(21.1 \%)$ & $2(10.5 \%)$ & $4(80 \%)$ & $1(20 \%)$ & 0 \\
\hline Streptomycin & $2(10.5 \%)$ & $3(15.8 \%)$ & $14(73.7 \%)$ & 0 & $1(20 \%)$ & $4(80 \%)$ \\
\hline Kanamycin & $1(5.3 \%)$ & $4(21.1 \%)$ & $14(73.7 \%)$ & 0 & $1(20 \%)$ & $4(80 \%)$ \\
\hline Imipenem & $18(94.7 \%)$ & $1(5.3 \%)$ & 0 & $4(80 \%)$ & 0 & $1(20 \%)$ \\
\hline
\end{tabular}




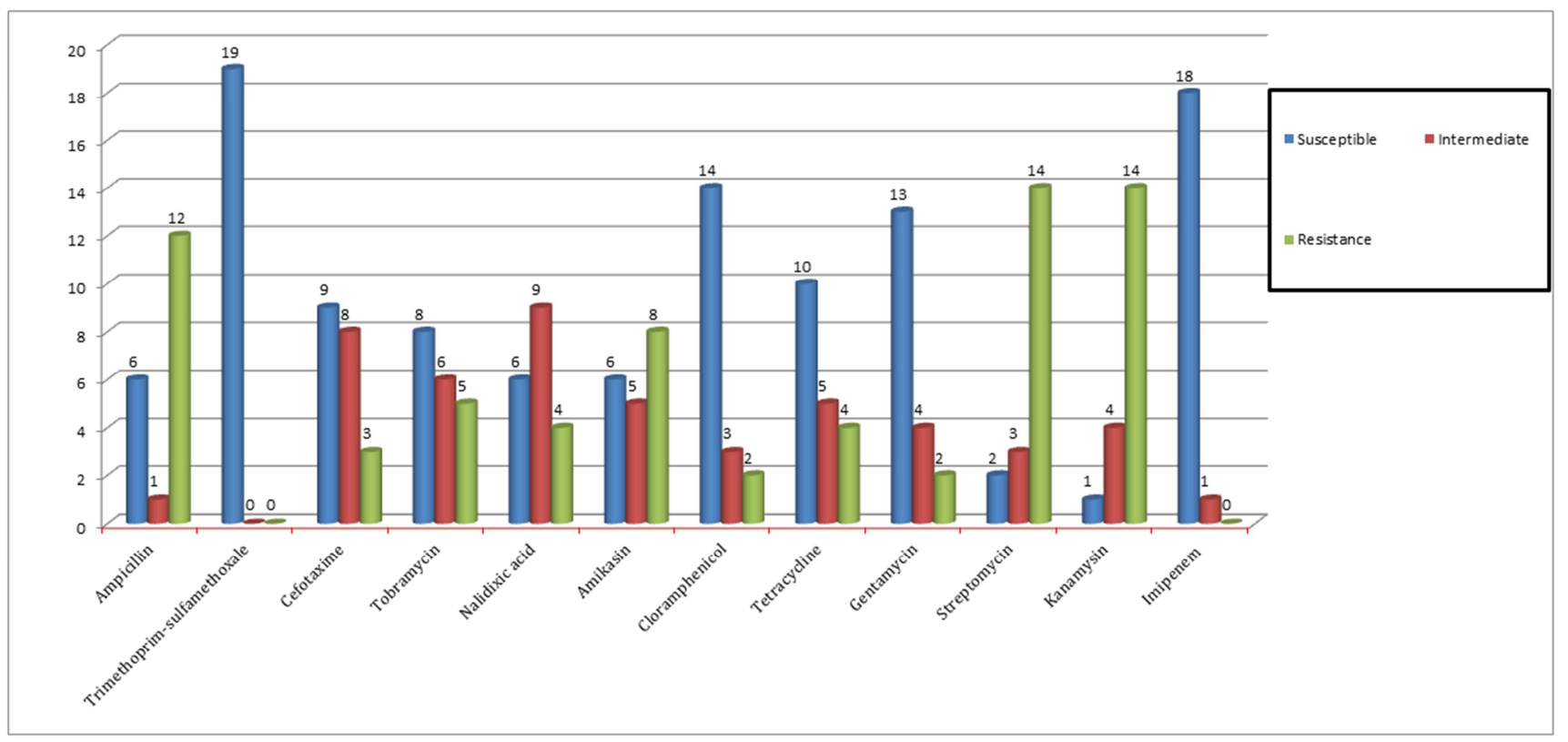

Figure 10. Antimicrobial-Resistance Profile of Klebsilla oxytoca isolates.

As shown on figure 10 Klebsilla oxytoca isolates showed antibiotics resistance to more than three antibiotics. Majority of them were resistance to Streptomycin, Kanamycin, Ampicillin and Amikacin respectively. In addition resistance was also observed to Cefotaxime, Tobramycin, Nalidixic acid, Amikacin, Chloramphenicol, Tetracycline, and Gentamycin. On the other hand majority of isolates were susceptible to Trimethoprim-sulfamethoxale, Imipenem, Chloramphenicol and Gentamycin [Figure 10].

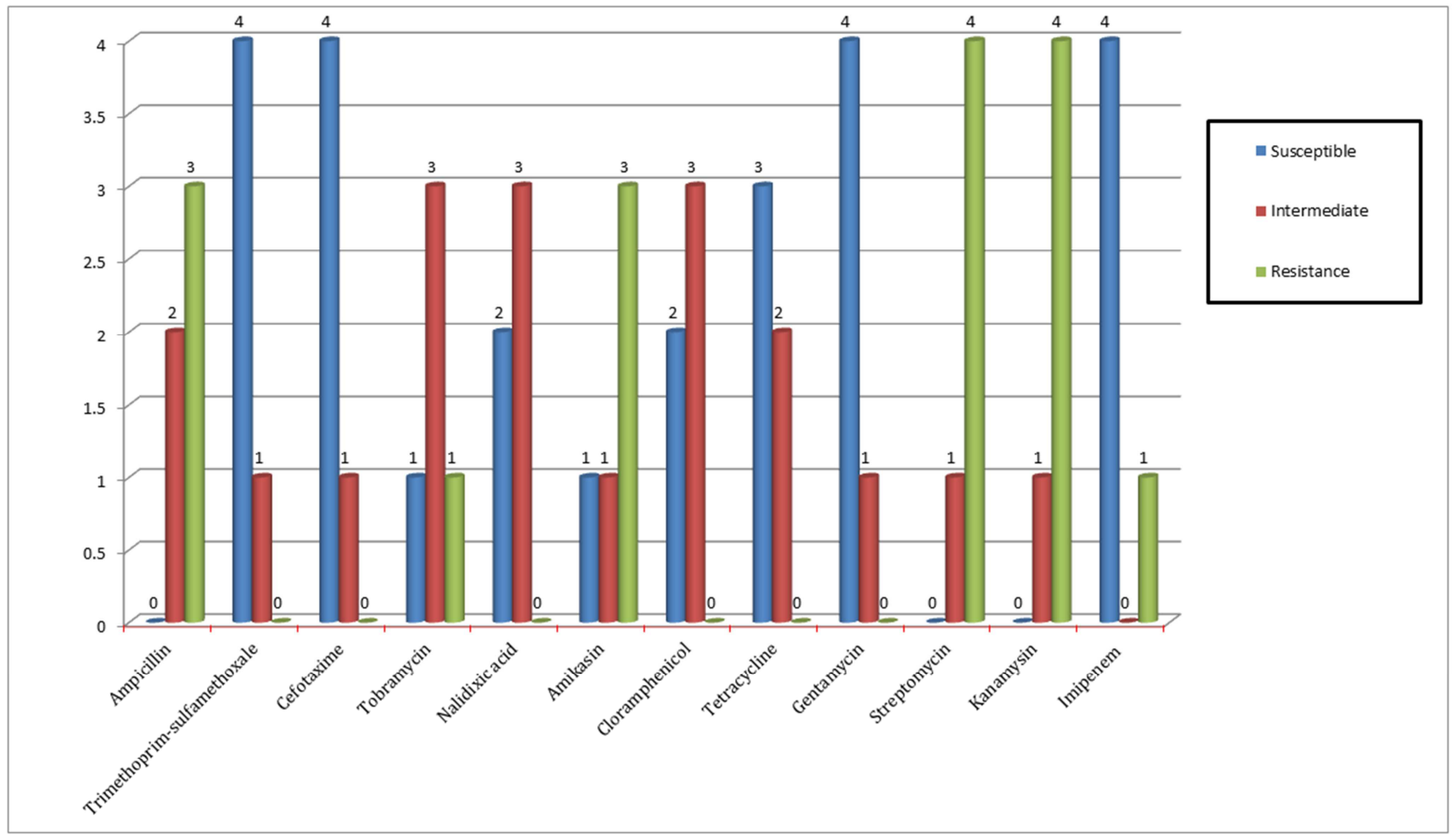

Figure 11. Antimicrobial-Resistance Profile of Klebsiella pneumoniae isolates.

As shown on figure 11 resistance was seen to Ampicillin, Amikacin, Streptomycin, and Kanamycin and few strains were also resistance to Tobramycin and Imipenem. On contrary to this susceptibility was observed on Trimethoprim- sulfamethoxale, Cefotaxime, Tetracycline, Gentamycin, Imipenem, Nalidixic acid, and Chloramphenicol [Figure 11] [Table 4]. 


\section{Discussion}

In this study, from a total of 100 food samples analyzed 19 (79\%) isolates were Klebsiella oxytoca and 5 (21\%) isolates were Klebsiella pneumoniae. In addition to Klebsiella spp., other enterobacteriaceae family members were also isolated from cheese and minced meat samples. Biofilms formed on different biotic and abiotic surfaces are responsible for many infections and can lead to significant health problems. Biofilm formation by Klebsiella species was detected using Microtiter Plate, Congo red agar and Tube methods. In our study all of Klebsiella pneumonia isolates were strong biofilm producers on TSB medium supplemented with $2 \%$ of glucose and variation on biofilm production was seen on TSB medium when glucose is not added. A study carried out by Seifi et al [29] demonstrate that $93.6 \%$ of $K$. Pneumoniae isolated from clinical samples were able to produce biofilms. However, in study conducted by Monirzadeh et al revealed majority of the isolates showed moderate and weak biofilm production i.e $40 \%(\mathrm{n}=35)$ displayed moderate activity, $30 \%$ $(n=26)$ demonstrated weak activity, $15 \% \quad(n=12)$ of the isolates showed strong biofilm activity, and 15\% $(n=12)$ showed no attachment to microtiter wells [30]. In another study conducted by $\mathrm{Oh}$ et al. [18], it was found that the importance of nutrient availability was demonstrated by the formation of biofilm on plastic in different media. Nowadays Klebsiella pneumoniae has got an attention around the hospital since it is an escalating human pathogen that that cause nosocomial infections and resistant to antibiotics and form biofilms [31].

The availability of nutrients, environment, geographical origin, types of specimen, surface adhesion characteristics, and genetic makeup of the organism have a tremendous role on biofilm formation [32]. Using tube and Congo red agar methods most Klebsiella pneumonia isolates were strong biofilm producer and slime factor positive respectively [Table 3]. Our result was consistent with other studies conducted by Osungunna and Onawunmi that revealed the Congo red agar method detected more biofilm-formers than the Tissue culture plate method with Klebsiella spp. As reported Osungunna and Onawunmi 50 isolates were biofilm-formers with $22 \%$ identified by the tissue culture plate method and $78 \%$ identified by the Congo red agar method [33]. Similarly in our study $74 \%$ and $60 \%$ of Klebsiella oxytoca and Klebsiella pneumoniae isolates were biofilm formers using Congo red agar method respectively. In another study conducted by Seifi et al [29] revealed that 93.6\% of Klebsiella pneumoniae isolates formed biofilms. Majority of Klebsiella pneumoniae (76.4\%) isolated from water samples also showed biofilm forming ability in vitro [34], which is in line with our findings that almost all isolates showed biofilm formation in all methods used for detections [Figure 9]. Using Microtiter Plate Method majority of our isolates were biofilm producers. In microtiter plate method, out of 19 Klebsiella oxytoca isolates 16 (84\%) were strong biofilm producers on TSB with $2 \%$ glucose after 24 hours of incubation. Variation on biofilm formation was seen when isolates are incubated on TSB media without adding glucose and biofilm production also varied on different incubation periods. Hence growth conditions such as substrates, incubation periods and other growth conditions may have an effect on biofilm production [Figure 2] [Table 2]. Like Klebsiella spp. other bacterial species such as Staphylococcus spp. showed biofilm formation when the TSB and BHI media are supplemented with glucose and sucrose [35]. Using tube method on TSB medium supplemented with $2 \%$ of glucose, out of 19 Klebsiella oxytoca isolates $68 \%(13 / 19)$ and $16 \%$ $(3 / 19)$ were strong and moderate biofilm producers after 24 hours of incubation. In tube and Congo red agar methods similarly majority of Klebsiella oxytoca isolates were strong biofilm producers and slime factor positive respectively [Table 3]. Biofilm formation is a complex process regulated by diverse factors, including the growth medium [10]. As indicated on many studies biofilm formation does not only confer survivability to the pathogen in the human hosts, but also persistence in the fluctuating environment such as variable $\mathrm{pH}$, temperature, carbon sources, and fluid flow. Biofilms can provide protection from environmental stresses such as $\mathrm{pH}$, osmotic shock, desiccation, and heat [36]. The existence of bacteria within this matrix substantially increases their resistance to environmental stress, antibiotics and detergents [37].

Antimicrobial susceptibility testing revealed that the 19 (100\%), $18(94.7 \%), 14(73.7 \%)$, and $13(68.4 \%)$ Klebsiella oxytoca isolates were susceptible to Trimethoprimsulfamethoxale, Imipenem, Chloramphenicol and Gentamycin respectively. Similarly $10(52.6 \%), 9(47.4 \%)$ and $8(42 \%)$ Klebsiella oxytoca isolates were also susceptible to Tetracycline, Cefotaxime and Tobramycin respectively [Table 4]. The resistance pattern of Klebsiella oxytoca to Streptomycin, Kanamycin and Ampicillin was, 14 (73.7\%), 14 (73.7\%) and 12 $(63.2 \%)$ respectively [Table 4]. A study conducted by Chakraborty et al. [38] showed that Klebsiella oxytoca was $100 \%$ resistant towards Ampicillin which was similar to our study even if few strains showed susceptibility to it. K. oxytoca revealed resistance to Nalidixic acid and Tetracycline by $75 \%$ and 50\% respectively [. Attachment along with biofilm formation among extensive drug resistant Klebsilla oxytoca isolates becomes an obstacle for eradication [39].

From total of 5 Klebsiella pneumoniae isolates majority of them i.e. $4(80 \%)$, were susceptible to Trimethoprimsulfamethoxale, Cefotaxime, Gentamycin and Imipenem. However, 4 (80\%) Klebsiella pneumoniae isolates were resistance for both Streptomycin and Kanamycin. In study conducted by Zhang et al. [40] resistance was seen in Streptomycin, Ampicillin and Amikacin which are similar with our study. The highest resistance rate was observed for ampicillin, followed by resistance to Tetracycline, Trimethoprim-sulfamethoxazole and Chloramphenicol [41]. Even if similar resistance was observed to Ampicillin, no resistance was observed to Tetracycline, Trimethoprimsulfamethoxale and Chloramphenicol in our study. In another study which was carried out by Khaertynov et al [42] all Klebsiella pneumoniae isolates were resistant to Ampicillin, 
Gentamycin and third-generation cephalosporins. However, all Klebsiella pneumoniae isolates were susceptible to Gentamycin in our study. Antibiogram of Klebsiella pneumoniae revealed resistance to Ampicillin, Amoxicillin, Ceftriaxone, Ciprofloxacin, Cotrimoxazole, Gentamicin, Nalidixic acid, Tetracycline by $100 \%, 94 \%, 50 \%, 37.5 \%$, $44 \%, 31 \%, 44 \%$ and $31 \%$ respectively [39]. Similarly resistance to Ampicillin, Gentamicin, Nalidixic acid and Tetracycline was observed in our study. As research findings revealed Klebsiella pneumoniae is a crucial cause of multidrug-resistant infections worldwide [43].

\section{Conclusion}

In this study, 19 Klebsiella oxytoca and 5 Klebsiella pneumonia isolates were obtained from cheese and minced meat samples. The majority of Klebsiella spp. were biofilm positive with variable degrees of production using different methods. In this study, strong biofilm production was detected when the growth medium is supplemented with other substrates such as glucose. The microorganism can form biofilms on different surfaces such as food and medical devices, becomes a potential source of contamination and creating food safety risks. In addition to this biofilms have a major role in the development of many nosocomial infections and leading to higher rates of device-related infections. Therefore, appropriate methods and strategies must be designed to prevent, control and combat biofilm formation. Among 19 Klebsiella oxytoca isolates, the highest rates of resistance were seen in streptomycin and Kanamycin followed by ampicillin. The majority of Klebsiella pneumoniae isolates were resistant to kanamycin and streptomycin followed by amikacin. Biofilms also pose serious problem health because of the increased resistance of biofilm-associated organisms to antimicrobial agents.

\section{Funding}

No fund is taken to conduct this paper.

\section{Availability of Data and Materials}

The data or information used to write this paper is available from the corresponding author upon request.

\section{Author Contributions}

The author was carried out all activities starting from collecting samples from the market to conducting all laboratory activities and to the final submission.

\section{Competing Interests}

I declare that there are no conflicts of interest in regard to the publication of this paper.

\section{Acknowledgements}

The authors would like to thank Gazi University biology staff members who helped me.

\section{References}

[1] Podschun R, Ullmann U (1998) Klebsiella spp. as nosocomial pathogens: epidemiology, taxonomy, typing methods, and pathogenicity factors. Clin Microbiol Rev, 11: 589-603.

[2] Darby A, Lertpiriyapong K, Sarkar U, Seneviratne U, Park DS, et al. (2014). Cytotoxic and Pathogenic Properties of Klebsiella oxytoca Isolated from Laboratory Animals. PLoS ONE 9 (7): e100542. doi: 10.1371/journal.pone.0100542.

[3] Singh C. L, Cariappa C. M. P., Kaur L. C. M. (2016). Klebsiella oxytoca: An emerging pathogen? Medical journal armed forces india 72, 5 9-61.

[4] Zollner-Schwetz I, Genauer C H, Joainig M, Weberhofer P, Gorkiewicz G, Valentin T, Hinterleitner TA, and Krause R (2008) Role of Klebsiella oxytoca in Antibiotic-Associated Diarrhea, Clinical Infectious Diseases 2008; 47: e74-8.

[5] Wei J, Wenjie Y, Ping L, Na W, Haixia R, Xuequn Z (2018). Antibiotic resistance of Klebsiella pneumoniae through $\beta$ arrestin recruitment-induced $\beta$-lactamase signaling pathway, Experimental and therapeutic medicine 15: 2247-2254.

[6] Ferreira RL, da Silva BCM, Rezende GS, Nakamura-Silva R, Pitondo-Silva A, Campanini EB, Brito MCA, da Silva EML, Freire CCM, Cunha AF and Pranchevicius MC (2019) High Prevalence of Multidrug-Resistant Klebsiella pneumoniae Harboring Several Virulence and $\beta$-Lactamase Encoding Genes in a Brazilian Intensive Care Unit. Front. Microbiol. 9: 3198. doi: 10.3389/fmicb.2018.03198.

[7] Brisse S, Fevre C, Passet V, Issenhuth-Jeanjean S, Tournebize $\mathrm{R}$, et al. (2009) Virulent Clones of Klebsiella pneumoniae: Identification and Evolutionary Scenario Based on Genomic and Phenotypic Characterization. PLoS ONE 4 (3): e4982. doi: 10.1371/journal.pone.0004982.

[8] Haryani Y, Noorzaleha A. S, Fatimah, A. B., Noorjahan, B. A., Patrick, G. B., Shamsinar, A. T., et al. (2007). Incidence of Klebsiella pneumoniae in street foods sold in Malaysia and their characterization by antibiotic resistance, plasmid profiling, and RAPD-PCR analysis. Food Control 18, 847853. doi: 10.1016/j.foodcont.2006.04.009.

[9] Chenga F, Li Z, Lana S, Liua W, Li X (2018). Characterization of Klebsiella pneumoniae associated with cattle infections in southwest China using multi-locus sequence typing (MLST), antibiotic resistance and virulence-associated gene profile analysis, Brazilian journal of microbiology 49S, 93-100, https://doi.org/10.1016/j.bjm.2018.06.004.

[10] Donlan RM, Costerton JW (2002) Biofilms: survival mechanisms of clinically relevant microorganisms. Clin Microbiol Rev 15: 167-193.

[11] Shi X, Zhu X (2009) Biofilm formation and food safety in food industries, Review 20, 407-413.

[12] Singh S, Singh S K, Chowdhury I, Singh R (2017) Understanding the Mechanism of Bacterial Biofilms Resistance to Antimicrobial Agents, The Open Microbiology Journal, 11, 53-62. 
[13] Schroll C, Barken K. B, Krogfelt K. A and Struve C., (2010) Research article Role of type 1 and type 3 fimbriae in Klebsiella pneumoniae biofilm formation, BMC Microbiology, 10: 179 http://www.biomedcentral.com/14712180/10/179.

[14] Zheng J, Lin Z, Chen C, Chen Z, Lin F, Wu Y, Yang S, Sun X, Yao W, Li D, Yu Z, Jin J, Qu D and Deng Q (2018) Biofilm Formation in Klebsiella pneumoniae Bacteremia Strains Was Found to be Associated with $\mathrm{CC} 23$ and the Presence of wcaG. Front. Cell. Infect. Microbiol. 8: 21. doi: 10.3389/fcimb.2018.00021.

[15] Hall-Stoodley L, Costerton J W, Stoodley P. (2004) Bacterial biofilms: from the natural environment to infectious diseases. Nat Rev Microbiol. 2004 Feb; 2 (2): 95-108.

[16] Ghasemian A, Mobarez A M., Peerayeh S N, Abadi A T B (2018). The association of surface adhesin genes and the biofilm formation among Klebsiella oxytoca clinical isolates, NMNI, 27, 36-39.

[17] Ribeiro SM, de la Fuente-Núñez C, Baquir B, Faria-Junior C, Franco OL, Hancock REW (2015). Antibiofilm peptides increase the susceptibility of carbapenemase-producing Klebsiella pneumoniae clinical isolates to -lactam antibiotics. Antimicrob Agents Chemother 59: 3906 -3912.

[18] Oh S, Chen P, Kang D (2007) Biofilm formation by Enterobacter sakazakii grown in artificial broth and infant formula on plastic surface. J Rapid Methods Automat Microbiol 15: 311-319.

[19] Iversen C, Lane M. Forsythe S J (2004) The growth profilethermotolerance and biofilm formation of Enterobacter sakazakii grown in infant formula milk. Lett Appl Microbiol38: 378-382.

[20] Kim H, Ryu J, Beuchat L (2006): Attachment of and biofilm formation by Enterobacter sakazakii on stainless steel and enteral feeding tubes: Appl Environ Microbiol 72 (9): 5846-5856.

[21] Najjuka C F, Kateete DP, Kajumbula HM, Joloba ML, Essack SY (2016). Antimicrobial susceptibility profiles of Escherichia coli and Klebsiella pneumonia isolated from outpatients in urban and rural districts of Uganda, BMC Res Notes (2016) 9: 235, DOI 10.1186/s13104-016-2049-8.

[22] Navon-Venezia S, Kondratyeva K, Carattoli A (2017) Klebsiella pneumoniae: a major worldwide source and shuttle for antibiotic resistance, Journal investing in science, FEMS Microbiology Reviews, 252-275.

[23] Adamo R, Margarit I. (2018). Fighting antibiotic-resistant Klebsiella pneumoniae with "sweet" immune targets. mBio 9: e00874-18. https://doi.org/10.1128/mBio.00874-18.

[24] Stepanovic S, Vukovic D, Hola V, Ventura GB, Djukic S, Irkovic Ruzika F (2007) "Quantification of biofilm in microtiter plates: Overview of testing conditions and practical recommendations for assessment of biofilm production by staphylococci “, Ap mis, 115: 891-9.

[25] Nasr R A, Abu Shady H M, Hussein H S (2012) Biofilm formation and presence of icaAD gene in clinical isolates of staphylococci, The Egyptian Journal of Medical Human Genetics, 13: 269-274.

[26] Christensen, G D, Simpson W A, Bisno A L, Beachey E H, Adherence of slime producing strains of Staphylococcus epidermidis to smooth surfaces", Infection and Immunity, 37:
318-26 (1982).

[27] Freeman D J, Falkiner F R, Keane C T (1989). New method for detecting slime production by coagulase negative staphylococci, Journal of Clinical Pathology, 42: 872-4.

[28] National Committee for Clinical Laboratory Standards (2003) Performance standards for antimicrobial susceptibility testing, 13th informational supplement, approved standard M100eS13 (M2). National Committee for Clinical Laboratory Standards, Wayne.

[29] Seifi K, Kazemian H, Heidari H, Rezagholizadeh F, Saee Y, Shirvani F, and Houri H (2016) Evaluation of Biofilm Formation Among Klebsiella pneumoniae Isolates and Molecular Characterization by ERIC-PCR, Jundishapur J Microbiol, 9 (1).

[30] Monirzadeh F, Shakibaie MR, Gholamrezazadeh M, Masoumi S (2018). Susceptibility of catheter-related Klebsiella pneumoniae strains to quaternary ammonium compounds under, Canadian Journal of Infection Control, 33 (4), 209-215.

[31] Khodadadian R, Rahdar HA, Javadi A, Safari M, Khorshidi A (2018). Detection of VIM-1 and IMP-1 genes in Klebsiella pneumoniae and relationship with biofilm formation, Microbial Pathogenesis, 115, 25-30, https://doi.org/10.1016/j.micpath.2017.12.036.

[32] Kokare C R, Chakraborty S, Khopade A N, Mahadik K R. (2009) Biofilm: importance and applications. Indian J Biotechnol.; 8: 159-168.

[33] Osungunna M O, Onawunmi G O (2018), Antibiotic resistance profiles of biofilm-forming bacteria associated with urine and urinary catheters in a tertiary hospital in Ile-Ife, Nigeria, Southern African Journal of Infectious Diseases, 33 (3): 80-85.

[34] Barati A, Ghaderpour A, Chew L. L, Bong C. W, Thong K. L, Chong V. C and Chai L. C (2016) Isolation and Characterization of Aquatic-Borne Klebsiella pneumoniae from Tropical Estuaries in Malaysia, Int. J. Environ. Res. Public Health, 13, 426.

[35] Mathur T, Singhal S, Khan S, Upadhyay D J, Fatma T, Rattan A. (2006) Detection of biofilm formation among the clinical isolates of staphylococci: An evaluation of three different screening methods. Indian J Med Microbiol; 24: 25-9.

[36] Kumar C G, Anand S K (1998). Significance of microbial biofilms in food industry: a review. International Journal of Food Microbiology, 42 (1), 9-27.

[37] Frank JF, Ehlers J, Wicker L (2003) Removal of Listeria monocytogenes and poultry soil-containing biofilm using chemical cleaning and sanitizing agents under static conditions. Food Protection Trends23: 654-663.

[38] Chakraborty S, Mohsına K, Sarker P K, Alam M. Z, Karım M I A, Sayem SM A (2016) Prevalence, antibiotic susceptibility profiles and ESBL production in Klebsiella pneumoniae and Klebsiella oxytoca among hospitalized patients, PERIODICUM BIOLOGORUM, 118, 1, 53-58.

[39] Ghasemian A, Mobarez AM, Peerayeh SN, Abadi ATM, Khodaparast S, Mahmood SS (2019) Expression of adhesin genes and biofilm formation among Klebsiella oxytoca clinical isolates from patients with antibiotic-associated haemorrhagic colitis, Journal of Medical Microbiology, 68 (7), https://doi.org/10.1099/jmm.0.000965. 
[40] Zhang S, Yang G, Ye Q, Wu Q, Zhang J, Huang Y (2018) Phenotypic and Genotypic Characterization of Klebsiella pneumoniae Isolated From Retail Foods in China, Front. Microbiol. 9: 289. doi: 10.3389/fmicb.2018.00289.

[41] Guo Y, Zhou H, Qin L, Pang Z, Qin T, Ren H, et al. (2016) Frequency, Antimicrobial Resistance and Genetic Diversity of Klebsiella pneumoniae in Food Samples. PLoS ONE 11 (4): e0153561. doi: 10.1371/journal.pone.0153561.

[42] Khaertynov KS, Anokhin VA, Rizvanov AA, Davidyuk YN,
Semyenova DR, Lubin SA and Skvortsova NN (2018) Virulence Factors and Antibiotic Resistance of Klebsiella pneumoniae Strains Isolated From Neonates With Sepsis. Front. Med. 5: 225. doi: 10.3389/fmed.2018.00225.

[43] Kidd TJ, Mills G, Sá-Pessoa J, Dumigan A, Frank CG, Insua JL, Ingram R, Hobley L, Bengoechea JA (2017) A Klebsiella pneumoniae antibiotic resistance mechanism that subdues host defences and promotes virulence, EMBO Mol Med (2017) 9: 430-447, DOI 10.15252/emmm.201607336. 